\title{
Hsp90 and reactive oxygen species regulate thermotolerance of rice seedlings via induction of heat shock factor A2 (OsHSFA2) and galactinol synthase 1 (OsGolS1)
}

\author{
Nong Thi Hue ${ }^{1^{\star}}$, Huong Thi Tran ${ }^{1^{\star}}$, Thuy Phan ${ }^{1}$, Junya Nakamura $^{1}$, Tomoe Iwata $^{2}$, \\ Keisuke Harano ${ }^{1}$, Yushi Ishibashi ${ }^{3}$, Takashi Yuasa ${ }^{3 \#}$, Mari Iwaya-Inoue ${ }^{3}$ \\ ${ }^{1}$ Graduate School of Bioresource and Bioenvironmental Sciences, Kyushu University, Fukuoka, Japan; \\ ${ }^{\#}$ Corresponding Author: yuasa@agr.kyushu-u.ac.jp \\ ${ }^{2}$ School of Agriculture, Kyushu University, Fukuoka, Japan \\ ${ }^{3}$ Department of Bioresource Sciences, Faculty of Agriculture, Kyushu University, Fukuoka, Japan
}

Received 30 January 2013; revised 19 February 2013; accepted 27 February 2013

\begin{abstract}
Heat stress induces expression of a set of thermotolerance-related genes in plants. We focused on rice (Oryza sativa L.) homologs of the gene family that encodes galactinol synthase (OsGolS), which is closely related to the Arabidopsis thaliana galactinol synthase (AtGolS) family whose expression is induced under various stresses. OsGolS1 was highly up-regulated compare to the level of OsGols2 in response to heat stress. Interestingly, OsGolS1 was also up-regulated by treatment with the Hsp90 inhibitor, geldanamycin (GDA). Expression profiles of OsGolS1 were correlated to those of OsHsfA2 under the GDA treatments. Treatment with GDA increased expression of OsHsfA2, but marginally increased or did not change OsHsfA1 expression. Notably, gel shift assay indicated that OsHsfA2 binds directly to OsGolS1 promoter region and that OsHsfA1 also binds to the promoter regions of OsHsfA2. Both OsHsfA2 and OsGolS1 were dramatically induced in response to heat stress. Accordingly, galactinol and raffinose contents in rice seedlings increased significantly following the induction of OsGolS1. Pre-treatment of rice seedlings with raffinose or GDA improved their thermotolerance. These results suggest that OsGolS1 plays an important role in response to heat stress, possibly via the transcription cascade of OsHsfA1-OsHsfA2 that leads to galactinol and raffinose accumulation, and that the increased content of these carbohydrates is a
\end{abstract}

\footnotetext{
*Two authors equally contributed to this study.
}

key response factor for rice seedlings to enhance thermotolerance.

Keywords: Galactinol Synthase; Geldanamycin; Heat Shock Factor; Heat Shock Protein; Oryza sativa

\section{INTRODUCTION}

High temperature stress is a dominant factor that restricts growth and yield of crops, and the effect of heat stress is progressively increasing due to the global warming. Previous genetic and biochemical studies have clarified the roles of heat shock proteins (Hsps) in both thermotolerance and protein stability [1-4]. Recently, it has been reported that the production of enzymes involving the biosynthesis of galactinol and of raffinose-family oligosaccharides (RFOs), and the resultant accumulation of RFOs, play pivotal roles in acquired tolerance of Arabidopsis to drought and temperature stresses [5]. Galactinol synthase (GolS), a key enzyme functioning at a rate-limiting step of RFOs biosynthesis, synthesizes galactinol from myo-inosytol and UDP-galactose which severs as a galactosyl donor to form raffinose, starchyose, and verbacose [6,7]. The accumulation of galactinol and RFOs appears to protect the photosynthetic apparatus when plants encounter oxidative stress, which can be generated by various environmental stresses [5].

The importance of galactinol and RFOs accumulation in plants under environmental stress raises a question of crosstalk between stress signaling and metabolic processes on the carbohydrate elevation. Recently, molecular and genetic research on A. thaliana have clarified that the transcription of the galactinol synthase genes AtGolS1 and AtGolS2 was induced by a combination of 
high-light and heat stress or by treatment with $\mathrm{H}_{2} \mathrm{O}_{2}$, and that oxidative stress stimulated the expression of heatshock factor A2 (AtHsfA2) [8,9]. Over-expression of AtHsfA2 resulted in increased AtGolS1 and AtGolS2 mRNA levels [5]. Notably, treatment of Arabidopsis with geldanamycin (GDA), an inhibitor of heat-shock protein 90 (Hsp90), significantly induced the expression of HsfA2 via dissociation of an Hsp90-HsfA complex [10]. Previous study on rice revealed that OsGolS1 and OsGolS2, the rice protein homologs of AtGolS1, share 72 and $71 \%$ amino acid sequence similarity to AtGolS1, respectively [11]. However, it remains unclear whether the expression of the gene for those proteins in rice is regulated by OsHsfA2 in response to environmental stresses.

To explore roles of OsGolS induction in the acquisition of heat tolerance by rice seedlings, and the possible involvement of OsHsfA2 in the gene induction, we determined the effects of heat stress on the gene expression, focusing on OsHsfA2 and OsGolS1. We also determined the effects of the Hsp90 inhibitor, geldanamycin, on the expression of OsHsfA2 and OsGolS1 and analyzed the interaction of OsHsfA2 with OsGolS1 promoter region. Carbohydrate metabolisms were monitored during the heat stress response by measuring the sugar contents of rice plants. To evaluate the involvement of OsGolS1 in the acquired thermotolerance of the rice plants, we examined effects of pretreatment with GDA and with raffinose on the growth and appearance during the recovering process after heat stress exposure. Based on the results of these studies, we hypothesize that the expression of OsGolS1 is regulated by OsHsfA2 which is induced by dissociation of the HSP90-OsHsfA1 complex under heat stress. The induction of OsGolS1 and the resultant galactinol and raffinose accumulation are crucial responses in the rice seedlings for the aquired thermotolerance.

\section{MATERIALS AND METHODS}

\subsection{Cultivation of the Rice Seedlings}

Oryza sativa L. cv. Hinohikari rice grains were treated by $70 \%$ ethanol for $15 \mathrm{~min}$, then soaked the grains in $0.2 \%$ sodium hypochlorite solution for $15 \mathrm{~min}$, followed by washing five times in distilled water. The disinfected rice grains were then incubated in distilled water at $30^{\circ} \mathrm{C}$ for 2 days, then moved to incubator at $25^{\circ} \mathrm{C}$ for 12 days using a cycle with $16 \mathrm{~h}$ light $\left(80 \mathrm{~W} \cdot \mathrm{m}^{-2}\right), 8 \mathrm{~h}$ dark, and $70 \%$ relative humidity.

\subsection{Time-Course Analysis after Heat Stress and Chemical Treatments}

As heat stress treatment, 14-day-old rice seedlings grown at $25^{\circ} \mathrm{C}$ were subjected to $52^{\circ} \mathrm{C}$ for $1,3,6,9 \mathrm{~h}$.
For the treatment with GDA, rice seedlings were treated by $50 \mu \mathrm{M}$ GDA for $3,6,8,12$, and $24 \mathrm{~h}$. Each sample consisted of $0.5 \mathrm{~g}$ of plant tissue collected at each point in time, and was immediately placed in liquid $\mathrm{N}_{2}$ and stored at $-80^{\circ} \mathrm{C}$ for use in subsequent analyses.

\subsection{RNA Extraction and Semi-Quantitative RT-PCR}

Total RNA was extracted from the plant tissue frozen in liquid $\mathrm{N}_{2}$ using the $\mathrm{SDS} / \mathrm{phenol} / \mathrm{LiCl}$ method as previously described [12]. RT-PCR was performed on a PC-816 thermal cycler (ASTEC Co., Fukuoka, Japan) in a $20-\mu \mathrm{L}$ reaction mixture containing $1 \mu \mathrm{L}$ cDNA sample, $200 \mu \mathrm{M}$ dNTPs, $400 \mathrm{nM} F$ (Forward)- and R (Reverse)primers (Table 1), and GoTaq Green Master Mix (Promega, MADISON WI, USA) in PCR reaction buffer under the following thermal cycle conditions: $94^{\circ} \mathrm{C}$ for 2 min; 27 - 30 cycles of $94^{\circ} \mathrm{C}$ for $10 \mathrm{sec}, 58^{\circ} \mathrm{C}$ for $10 \mathrm{sec}$, and $72^{\circ} \mathrm{C}$ for $30 \mathrm{sec}$; a final extension at $72^{\circ} \mathrm{C}$ for $1 \mathrm{~min}$; and a final hold at $16^{\circ} \mathrm{C}$ for $99 \mathrm{~min}$. DNA was separated in $1.5 \%$ agarose gels and stained with ethidium bromide, then visualized using FluorChem (Alpha Innotech, San Leandro, CA, USA).

\subsection{Construction of pMal-OsHsfA1 and pMal-OsHsfA2}

To prepare maltose binding protein (MBP)-fused OsHsfA1 and OsHsfA2, we constructed pMal-OsHsfA1 and -OsHsfA2. PCR fragments encoding full length OsHsfA1 and OsHsfA2 were amplified with KOD Plus DNA polymerase (TOYOBO, Tokyo, Japan), rice cDNA, and a gene-specific primer set (OsHsfA1-FL and OsHsfA2-FL) (Table 1), and then digested with BamHI and SalI. The resultant fragments were ligated into BamHI-SalI sites of pMal-c (New England Biolabs, Inc., Ipswich, MA) by DNA Ligation Kit v. 2 (Takara Bio Inc., Tokyo, Japan). The cloned OsHsfA1 and OsHsfA2 cDNAs were confirmed by DNA sequencing. The recombinant proteins, MBP, MBP-OsHsfA1, and MBPOsHsfA2, were expressed in Escherichia coli cells (BL21DE) containing pMal-c empty vector, pMalOsHsfA1 and pMal-OsHsfA2 grown over night at $37^{\circ} \mathrm{C}$ in $\mathrm{LB} / \mathrm{Amp}$ medium in the presence of $0.5 \mathrm{mM}$ IPTG.

\subsection{Protein Expression and Purification of Recombinant Proteins}

The recombinant proteins were purified from the $E$. coli cells with Amylose Resin column (NewEngland Lab Inc.), as described in the manufacturer's instruction manual with minor modifications [13]. Protein concentrations were determined by measuring $\mathrm{OD}_{595}$ with a Bio Rad protein assay kit (Bio Rad, Hercules, CA, USA), using $1 \mathrm{mg} \cdot \mathrm{mL}^{-1}$ bovine serum albumin as a standard. 
Table 1. Gene specific oligo DNA primers used.

\begin{tabular}{|c|c|c|}
\hline Gene name & $\begin{array}{l}\text { Accession } \\
\text { No./(RGI) }\end{array}$ & Nucleotide Sequences \\
\hline \multirow[t]{2}{*}{ OsHsfA1 } & AK100430 & Fwr: GGTGGATCCTTGCAGAGGTCCCAGCTAAC \\
\hline & (Os03g0854500) & Rev: TCCTGTATGGTGTGATGACAGGAGGCCCT \\
\hline \multirow[t]{2}{*}{ OsHsfA1- FL } & & Fwr: GGATGGATCCATGGAGGCCGCCGTTGCTGCTGCGGC \\
\hline & & Rev: TCCTAGTCGACCTCATCATCCTGTATGGTGTGATGA \\
\hline \multirow[t]{2}{*}{ OsHsfA2 } & AY344488 & Fwr: TATCTCGGAATCCGAACAAGTCAACTTGG \\
\hline & (Os10g28340) & Rev: TCAAGCTTGTTTCCTCAAATCCTTGGAATG \\
\hline \multirow[t]{2}{*}{ OsHsfA2-FL } & AY344488 & Fwr: AGGGGGATCCATGGACCCGGCGGCGGCGGGCATTGT \\
\hline & (Os10g28340) & Rev: CAAAGTCGACTCTATTTTGGACTAGTAGAGC \\
\hline \multirow[t]{2}{*}{ Pro-OsHsfA2(10-190) } & & Fwr: GTTTGACCTCGAAAGGGAAAGCAGAAG \\
\hline & & Rev: CTCCCGCCTTTCACAACCAAGAACCCA \\
\hline \multirow[t]{2}{*}{ Pro-OsHsfA2(200-400) } & & Fwr: CСТCTCTCCACTCTCCAGAAGCTTCTC \\
\hline & & Rev: AATGGTGGGGATGAAACGAAGCGAGAA \\
\hline \multirow[t]{2}{*}{ OsGolS1 } & AK071812 & Fwr CCTTGACGCCGACATCCAGGTGTTCGACAA \\
\hline & (Os03g0316200) & Rev: GTCGTTGTAGACGTCCCACCACTTCTTGAC \\
\hline \multirow[t]{2}{*}{ Pro-OsGolS1 } & & Fwr: CAAGATGGAAGTACACAGTCAAATCCC \\
\hline & & Rev: AATAGGAAACGGAGGGGGCGAAATGGT \\
\hline \multirow[t]{2}{*}{ Pro-OsHsfA3 } & Os02g0527300 & Fwr: TTCGGCCAGCCACCGCCTCCCCTCCCG \\
\hline & & Rev: GGCTTCGGGTTCGAAACCGACATACTG \\
\hline \multirow[t]{2}{*}{ OsGolS2 } & D26537 & Fwr: CTCTACTTCAACGCCGGCTAGTTCG \\
\hline & $(\mathrm{Os} 07 \mathrm{~g} 0687900)$ & Rev: TGTAGCTATCAAGACTTGACGGCGGG \\
\hline \multirow[t]{2}{*}{$\beta$-Tubulin } & AK243618 & Fwr: ACTTCTTCATGGTCGGCTTCGCCCCGCT \\
\hline & $(\mathrm{Os} 03 \mathrm{~g} 01530)$ & Rev: ATCCTCGTATTCACCCTCGTCGTCGGCGGT \\
\hline
\end{tabular}

RGI, rice gene indics were referred to The Rice Annotation Project (RAP); URL http://rapdb.dna.affrc.go.jp/

\subsection{Gel-Shift Assay}

Rice genomic DNA was prepared from 14-day-old rice seedlings by CTAB method. Promoter regions (about 200 bp) of OsGolS1, OsHsfA2 (10 - 190), (200 - 400) and OsHsfA3 were amplified by PCR in a $50-\mu \mathrm{L} 1 \times$ GoTaq Green Master Mix reaction mixture containing $100 \mathrm{ng}$ rice total genomic DNA, $400 \mathrm{nM} \mathrm{F}$ - and R-promoter specific primers sets (Table 1), under the following thermal cycle conditions: $94^{\circ} \mathrm{C}$ for 2 min; 35 cycles of $94^{\circ} \mathrm{C}$ for $10 \mathrm{sec}, 58^{\circ} \mathrm{C}$ for $10 \mathrm{sec}$, and $72^{\circ} \mathrm{C}$ for $20 \mathrm{sec}$; a final extension at $72^{\circ} \mathrm{C}$ for $5 \mathrm{~min}$; and a final hold at $16^{\circ} \mathrm{C}$ for $99 \mathrm{~min}$. For a gel-shift assay, a $10 \mu \mathrm{L}$ of reaction mixture containing $10 \mathrm{mM}$ Tris- $\mathrm{HCl}$ pH7.5, $50 \mathrm{mM} \mathrm{NaCl}, 1 \mathrm{mM}$ $\mathrm{MgCl}_{2}, 0.1 \% \beta$-mercaptoethanol, $100 \mathrm{ng}$ promoter DNA (OsGolS1 or OsHsfA3) and 1 to $3 \mu \mathrm{g}$ recombinant protein (MBP, MBP-OsHsfA1 or MBP-OsHsfA2) was incubated at $25^{\circ} \mathrm{C}$ for $30 \mathrm{~min}$, then separated in $2 \%$ agarose gels [14]. PCR product coding a promoter region of OsHsfA3, which is reported to be induced under cold stress but not heat stress, was used as a negative control $[13,15]$. The DNA and DNA-protein complexes stained with ethidium bromide were visualized by FluorChem imager (AlphaInnotech, San Leandro, CA, USA).

\subsection{Measurement of Endogenous $\mathrm{H}_{2} \mathrm{O}_{2}$ Levels}

The endogenous $\mathrm{H}_{2} \mathrm{O}_{2}$ content was measured according to the method of Veljovic-Jovanovic et al. [16]. Briefly, plant tissue $(0.5 \mathrm{~g})$ was ground in $0.5 \mathrm{~mL}$ of 0.2 $\mathrm{M}$ perchloric acid until completely homogenized, using a mortar and pestle. $2 \mathrm{~mL}$ of the homogenate was then centrifuged at $13,000 \times \mathrm{g}$ for $15 \mathrm{~min}$. The supernatant was transferred into $1.5 \mathrm{~mL}$ plastic tube with $0.5 \mathrm{~mL}$ of $4 \mathrm{M}$ potassium hydroxide then centrifuged at $13,000 \times \mathrm{g}$ for 3 min. The supernatant was tested using the peroxidase enzyme reaction. We created a compound solution by mixing $60 \mathrm{~mL}$ of $12 \mathrm{mM} m$-dimethylamino benzoic acid with $0.5 \mathrm{~mL}$ of $1.3 \mathrm{M}$ 3-methyl-2-benzothiazole nonhydrazone hydrochloride salt-hydrate. We placed $1430 \mu \mathrm{L}$ of the compound solution in a glass cuvette then added $50 \mu \mathrm{L}$ of the supernatant and $20 \mu \mathrm{L}$ of peroxidase $(0.25$ Unit). The solution was analyzed using a U-1800 spectrophotometer (Hitachi, Tokyo, Japan). The absorbance as a result of the reaction was measured after $5 \mathrm{~min}$ in the U-1800 spectrophotometer at $590 \mathrm{~nm}$. We created a standard curve using $0.125,0.25,0.50$, and $1.0 \mu \mathrm{M} \mathrm{H}_{2} \mathrm{O}_{2}$ solutions. Experiments were repeated for the three batches of 14-day-old rice seedlings which were grown at the same condition.

\subsection{Measurements of the Contents of Galactinol, Rafinose and Other Carbohydrates}

Plant tissue $(0.5 \mathrm{~g})$ was ground to become a fine powder in liquid $\mathrm{N}_{2}$ and then homogenized with $5 \mathrm{~mL}$ of $80 \%$ ethanol (v/v) using a mortar and pestle. $300 \mu \mathrm{L}$ of 15 $\mathrm{mM}$ lactose was added to the homogenate as a control. The homogenate was boiled for $30 \mathrm{~min}$ at $80^{\circ} \mathrm{C}$ and then centrifuged for $15 \mathrm{~min}$ at $10,000 \times \mathrm{g}$. Extracts were dried and dissolved in $0.5 \mathrm{~mL}$ of distilled water. We then added $0.5 \mathrm{~mL}$ of Dowex (Muromachi Gagaku Kokyo, Japan) to adsorb the suspension at $4^{\circ} \mathrm{C}$ for $60 \mathrm{~min}$. Galactinol and 
other sugars were analyzed by high-performance ionexchange chromatography (LC-10A system, Shimazu, Tokyo, Japan) and refractive index detector (RID10A, Shimazu) and an $\mathrm{NH}_{2} \mathrm{P}-50$ 4E column (Shodex, Tokyo, Japan), which is a polyamine-bonded silica-based amino column. The flow rate was set to $0.7 \mathrm{~mL} \cdot \mathrm{min}^{-1}$, with acetonitrile- $\mathrm{H}_{2} \mathrm{O}(75: 25 \mathrm{v} / \mathrm{v})$ as the carrier, according to the method of Ishibashi et al. [17]. All measurements were repeated for each extracts from the three batches of plants.

\subsection{Heat Stress Tolerance Test}

A batch of 14-day-old rice seedlings grown at $25^{\circ} \mathrm{C}$ was pre-treated by $5 \mu \mathrm{M}$ geldanamycin for $12 \mathrm{~h}$, and the other batch of rice seedlings was pre-treated by $50 \mathrm{mM}$ raffinose for $12 \mathrm{~h}$. After the pre-treatment, the rice seedlings were subjected to high temperature at $52^{\circ} \mathrm{C}$ for $3 \mathrm{~h}$, then moved to control condition. The appearance of the rice seedlings were observed at 7 days after heat stress.

\section{RESULTS}

\subsection{OsHsfA1, OsHsfA2 and OsGolS1 mRNA Levels Increased under Heat Stress}

To analyze the expression of heat-inducible genes, rice seedlings were subjected to high temperature as sublethal level at $52^{\circ} \mathrm{C}$ for $3 \mathrm{~h}$. The expression of OsGolS1 increased drastically in response to the severe heat stress compared to expression levels in the control (Figure 1) and remained at high level when the rice seedlings were continuously exposed to heat stress (data not shown). The expression of OsHsfA1 remained at a constant level whereas OsHsfA2 was highly up-regulated under heat stress (Figure 1). The mRNA levels of OsGols2 increased slightly after heat stress. However, the mRNA levels of OsGolS1 were significantly higher than those of OsGolS2, indicating that OsGolS1 plays a major role in the biosynthetic pathway of galactinol and the heat-stress response.

\subsection{GDA Treatments Induced the Expression of OsHsfA2 and OsGolS1}

Previous studies on Arabidopsis revealed that GDA, an Hsp90 inhibitor, disassembles Hsp90-HsfA1 complexes, leading to the activation of HsfA1; this activated form of HsfA1 is necessary for the subsequent up-regulation of heat-inducible genes $[10,18]$. These findings prompted us to analyze the effects of GDA on the expression of OsHsfA2 and OsGolS1. Interestingly, under GDA treatments, the expression of OsHsfA2 and OsGolS1 increased remarkably (Figure 2). The induction of OsHsfA2 was observed at $3 \mathrm{~h}$ after GDA treatment followed by up-regulation of OsGolS1 at $8 \mathrm{~h}$, and then the

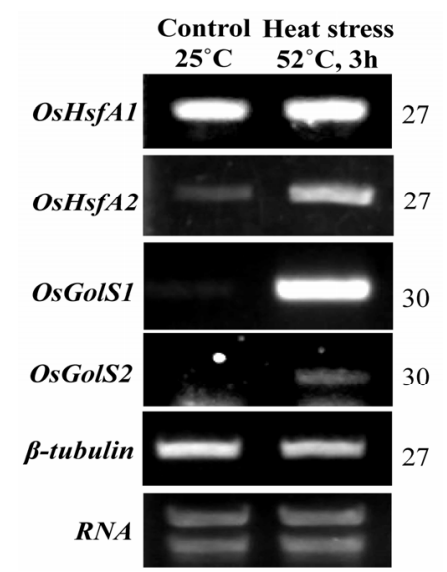

Figure 1. Expression profiles of OsHsfA1, OsHsfA2, OsGolS1 and OsGolS2 under control and heat stress condition. 14-day-old rice seedlings grown at $25^{\circ} \mathrm{C}$ were exposed to heat stress at $52^{\circ} \mathrm{C}$ for $3 \mathrm{~h}$. Controls were kept at $25^{\circ} \mathrm{C}$. $\beta$-tubulin and mRNA indicated internal control. Representative images are shown from one of three experiments.

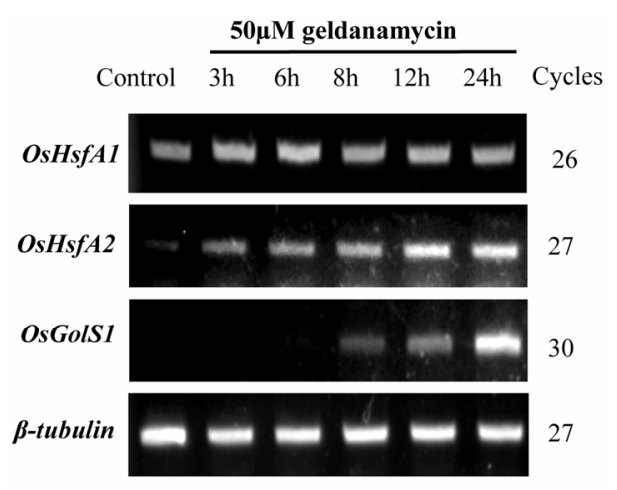

Figure 2. Time course analysis for the expression of OsHsfA1, OsHsfA2 and OsGolS1 under Hsp90 inhibitor (GDA) treatment. 14-day-old rice seedlings grown at $25^{\circ} \mathrm{C}$ were treated by $50 \mu \mathrm{M}$ GDA for $3,6,8,12$ and 24 h. $\beta$-tubulin indicated internal control. Representative images are shown from one of three repeated experiments.

expression of both genes increased up to $12 \mathrm{~h}$ in the case of OsHsfA2 and $24 \mathrm{~h}$ in the case of OsGolS1. In contrast, we observed no significant change in OsHsfA1 mRNA levels (Figure 2). These results indicate that OsGolS1 induction was correlated with the expression profile of OsHsfA2 which was induced under the GDA treatment.

\subsection{OsHsfA2 Binds to Heat-Shock Elements (HSE) in the OsGolS1 Promoter Region}

To determine whether OsHsfA2 is involved in regulation of OsGolS1 expression, nucleotide sequence motiv- 
es related to heat shock elements in the promoter region of OsGolS1 and their interaction with OsHsfA2 were examined (Figure 3). The inspection of the putative promoter region of OsGolS1 revealed the TATA boxproximal 5' flanking region of OsGolS1 gene indicates that several HSE-like consensus motives potentially associating with HSFs are identified at positions indicated by \#1-10 (Figure 3(a)). Particularly, position \#4 has a sequence closely similar to the efficient HSE consensus [nGAAnnTTCnnGAAn] and other \#1-3 and \#5-10 also contain the core consensus motives of [nGAAn] and [nTTCn] (Figure 3(a)) [19]. In contrast, an amplified PCR fragment of OsHsfA3 promoter region contains only 3 sites of the core consensus motives in "TTCGAACCCGAA" closed to TATA-box. A purified MBP-OsHsfA2 purified from $E$. coli was confirmed at a relative molecular mass of approximately $86 \mathrm{kDa}$ by SDS-PAGE (Figure 3(b)). Interaction of the MBP-OsHsfA2 with the promoter regions of OsGolS1 and OsHsfA3 was determined by gel-shift assay. The two shifted bands indicated that MBP-OsHsfA2 formed a complex with the OsGolS1 promoter region, while there no shifted band was observed with regard to a mixture of MBP-OsHsfA2 and OsHsfA3 promoter region (Figure 3(c)). In addition, no mobility shift according to the DNA-protein complex was observed in the mixture of MBP with the OsGolS1 promoter region. An increase of the OsHsfA2 protein amounts in the assay mixtures resulted in the uppershifted bands of the OsHsfA2/DNA-complex, indicating that the OsGolS1 promoter contains multiple HSE consensus sites or different OsHsfA2-binding affinities of the HSE consensus-like motives dependent on OsHsfA2

\section{a}

5'-Primer $\rightarrow$

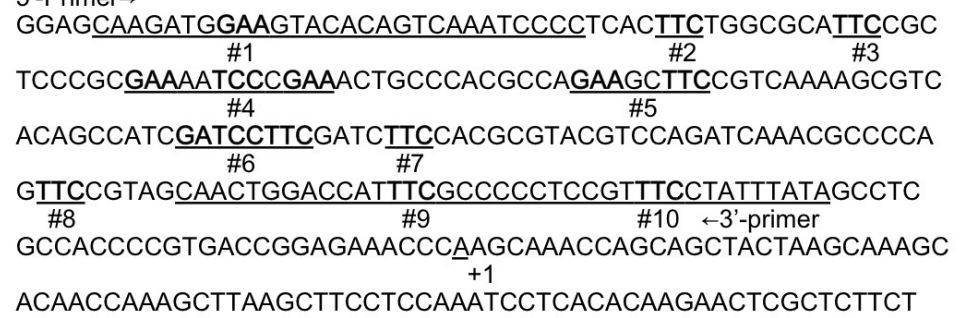

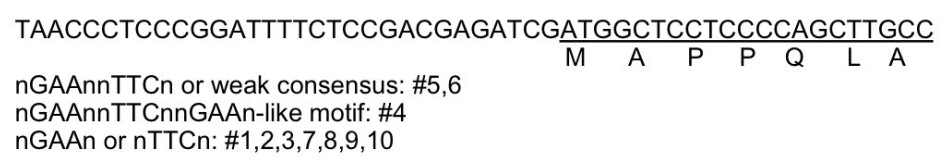

nGAAn or nTTCn: \#1,2,3,7,8,9,10
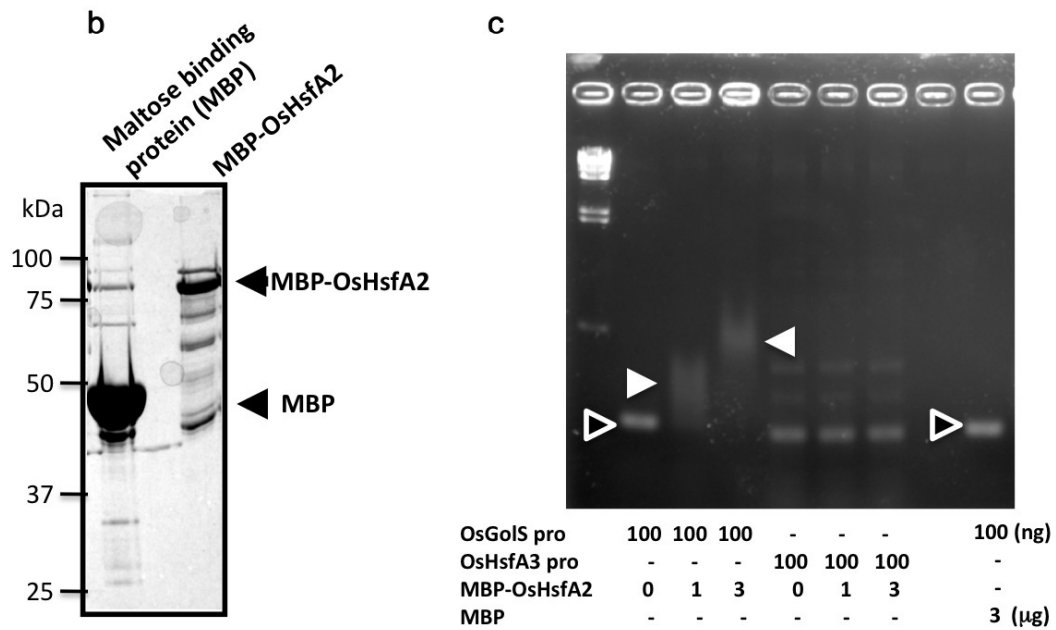

Figure 3. Binding of OsHsfA2 to the OsGolS1 promoter fragment. (a) The DNA sequence of promoter upstream region of OsGolS1. The HSE-related sequences are indicated by the numbers from \#1 to 10, \#4 represents an efficient HSE consensus. TATA-box and a start codon are underlined. The sequence of DNA fragment was cloned using 5'- and 3'-primers indicated in the sequence, which used for gel-shift assay; (b) The purified MBP-OsHsfA2 protein was confirmed by SDS-PAGE; (c) Interaction between MBP-OsHsfA2 and the OsGolS1 promoter. Closed triangles indicate the mobility shift of complexes of MBP-OsHsfA2 protein and OsGolS1 promoter fragment. Open triangles indicate protein-unbound DNA fragments without mobility shift. MBP and the promoter region of OsHsfA3 were used as negative control. The molecular size maker was $\lambda$ Hind III. 
protein levels. These data revealed the binding activity of the recombinant OsHsfA2 to the promoter region of OsGolS1 at specific HSEs, suggesting that OsHsfA2 is involved in regulation of OsGolS1 expression in response to heat stress.

\subsection{OsHsfA1 Binds to the Promoter Regions of OsHsfA2}

To determine whether OsHsfA1 is involved in transcriptional regulation of OsHsfA2 and/or OsGolS1, interactions of the promoter regions of OsHsfA2 and OsGolS1 with OsHsfA1 were examined by gel shift assay (Figure 4). A recombinant MBP-OsHsfA1 purified from $E$. coli was confirmed at the relative molecular mass of approximately $100 \mathrm{kDa}$ by SDS-PAGE (Figure 4(a)). Interaction of the MBP-OsHsfA1 with the promoter regions of OsHsfA2, OsGolS1 and OsHsfA3 was compared (Figures 4(b) and (c)). Two regions from -10 to -190 , and from -200 to -400 at upstream of the TATA box of OsHsfA2 gene were amplified by PCR as probe. Single and significant band shifts of both Pro-OsHsfA2 (10 $190)$ and $(200$ - 400) in the presence of MBP-OsHsfA1 indicated that MBP-OsHsfA1 formed a complex with the OsHsfA2 promoter regions, while there no shifted band was observed with regard to a mixture of MBP-OsHsfA1 and OsHsfA3 promoter region (Figure 4(b)). In addition, no mobility shift according to the DNA-protein complex was observed in the mixture of MBP with the OsHsfA2 promoter regions. An increase of the OsHsfA1 protein amounts in the assay mixtures resulted in upper-shift and increased signal of the OsHsfA1/DNA-complex, indicating that the OsHsfA2 promoter contains multiple HSE consensus sites. As same as the OsHsfA2 promoter fragments, significant signals according to the DNA-protein complex composed of MBP-OsHsfA1 and OsGolS1 promoter fragment were detected as mobility shift in the mixture of MBP-OsHsfA1 with the OsGolS1 promoter fragment, while the bands of the protein-unbound OsGolS1 promoter fragment disappeared in the presence of MBP-OsHsfA1 (Figure 4(c)). The data revealed that the OsHsfA1 protein binds to the promoter regions of both OsHsfA2 and OsGolS1, suggesting that OsHsfA1 is involved in transcriptional regulation at upstream of OsHsfA2 and OsGolS1 in response to heat stress.

\subsection{Endogenous $\mathrm{H}_{2} \mathrm{O}_{2}$ Levels in Rice Seedling during Heat Stress}

It has been convincingly demonstrated that heat stress

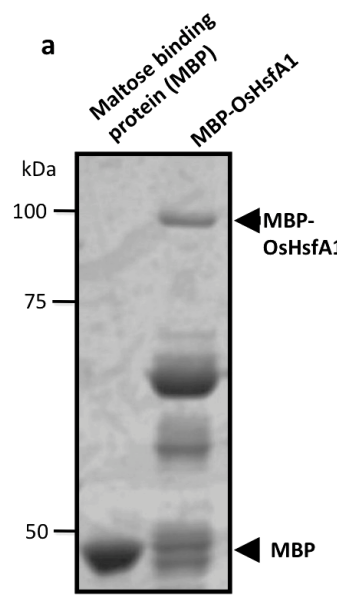

b

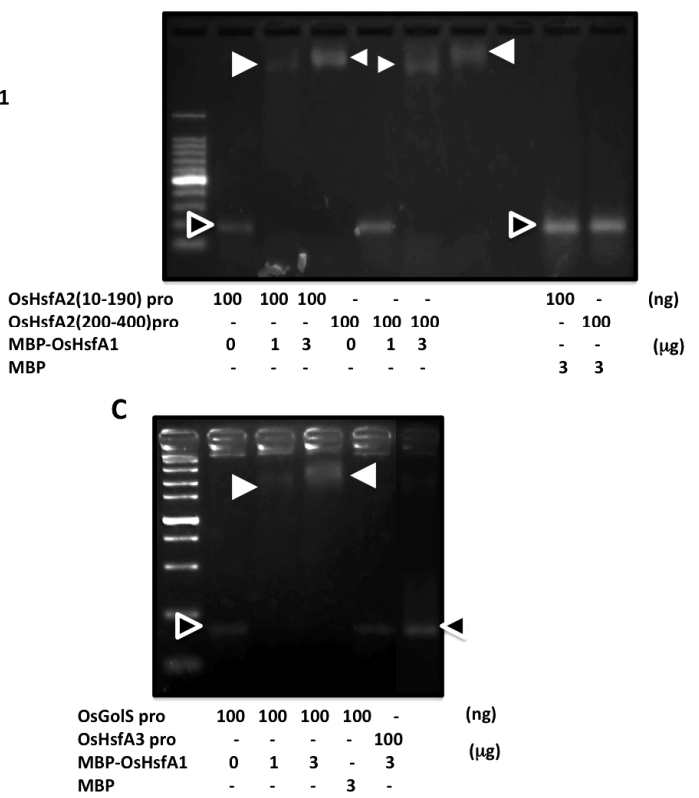

Figure 4. Interactions of OsHsfA1 to the OsHsfA2 and OsGolS1 promoter fragments. (a) The purified MBP-OsHsfA1 protein was confirmed by SDS-PAGE; (b) Interaction between MBPOsHsfA1 and the promoter fragments of OsHsfA2 (10 - 190) and (200 - 400); (c) Interaction between MBP-OsHsfA1 and OsGolS1 promoter fragment. Closed triangles indicate the mobility shift of the complex of MBP-OsHsfA1 and promoter fragments. Open triangles indicate protein-unbound DNA fragments without mobility shift. MBP and the promoter region of OsHsfA3 were used as negative controls. 
increases the production of reactive oxygen species (ROS) such as superoxide anions $\left(\mathrm{O}_{2}^{-}\right)$, hydrogen peroxide $\left(\mathrm{H}_{2} \mathrm{O}_{2}\right)$, and hydroxyl radicals $\left(\mathrm{OH}^{-}\right)$in plant cells [20]. When rice seedlings were subjected to heat stress, the endogenous $\mathrm{H}_{2} \mathrm{O}_{2}$ level in the rice seedlings increased significantly $\left(\mathrm{p}<0.01\right.$ ), from $0.24 \mu \mathrm{mol} \cdot \mathrm{g}^{-1}$ at $0 \mathrm{~h}$ (control level) to $0.43 \mu \mathrm{mol} \cdot \mathrm{g}^{-1}$ at $3 \mathrm{~h}$, and then decreased slightly to remain stable at approximately $0.35 \mu \mathrm{mol} \cdot \mathrm{g}^{-1}$ from $6 \mathrm{~h}$, which was not significantly different from the control level (Figure 5).

\subsection{Galactinol Was Accumulated by Heat Stress Treatments}

To examine whether the OsGolS1 induction was correlated with galactinol accumulation, we treated 14-dayold rice seedlings with heat stress to measure the profiles of galactinol and other carbohydrates with the time course based on the induction of OsGolS1 which was described in the method. Under heat stress, the galactinol content increased significantly $(\mathrm{p}<0.01)$, from 0.44 $\mathrm{mg} \cdot \mathrm{gFW}^{-1}$ at $0 \mathrm{~h}$ to $1.6 \mathrm{mg} \cdot \mathrm{gFW}^{-1}$ after $9 \mathrm{~h}$, which represents an increase to approximately four times the original value (Figure 6(a)). Accordingly, raffinose content also increased significantly $(\mathrm{p}<0.01)$ from $0.33 \mathrm{mg} \cdot \mathrm{gFW}^{-1}$ at $0 \mathrm{~h}$ or control level to $1.74 \mathrm{mg} \cdot \mathrm{gFW}^{-1}$ at $9 \mathrm{~h}$ which was slightly higher than the level of galactinol at $9 \mathrm{~h}$ (Figures 6(a), (b)). The sucrose content was also highly accumulated from $3.25 \mathrm{mg} \cdot \mathrm{gFW}^{-1}$ at $0 \mathrm{~h}$ to $9.03 \mathrm{mg} \cdot \mathrm{gFW}^{-1}$ (nearly three times the initial level) by $9 \mathrm{~h}$ (Figure 6(c)). Glucose and fructose levels did not change significantly during the heat stress treatments (Figures 6(d) and (e)).

\subsection{Pre-Treatment with GDA or Raffinose Enhanced the Thermotolerance of the Rice Seedlings}

To determine the effects of GDA and raffinose pre-

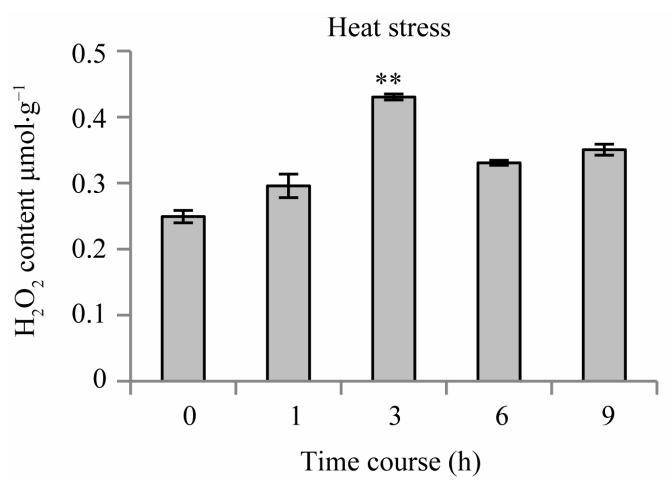

Figur 5. Changes in endogenous levels of $\mathrm{H}_{2} \mathrm{O}_{2}$ in rice seedlings under heat stress. For the heat stress treatment, 14-day-old rice seedlings grown at $25^{\circ} \mathrm{C}$ were subjected to heat stress at $52^{\circ} \mathrm{C}$ for $1,3,6$ and $9 \mathrm{~h} ; 0 \mathrm{~h}$ represents control. Bar is standard error $(n=3,(* *): p$ $<0.01)$. FW, Fresh Weight. (a)

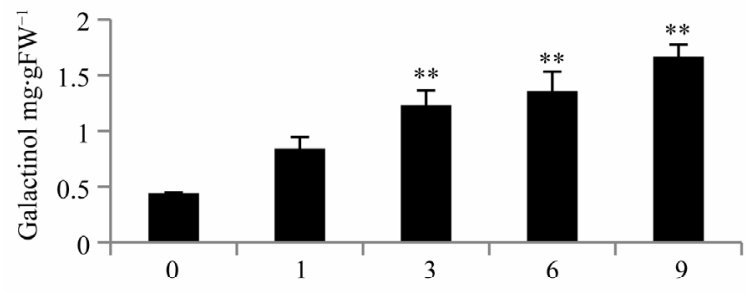

(b)
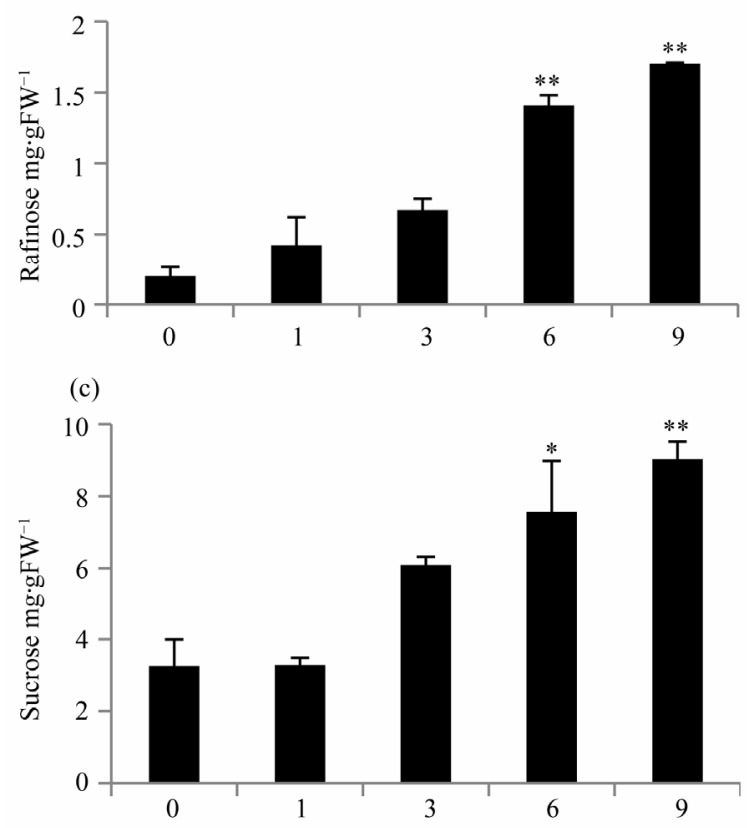

(d)

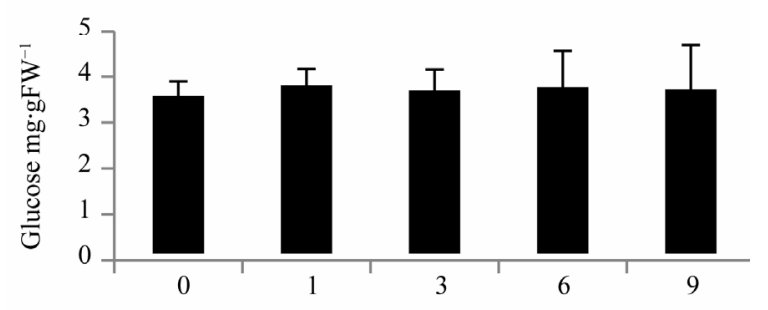

(e)

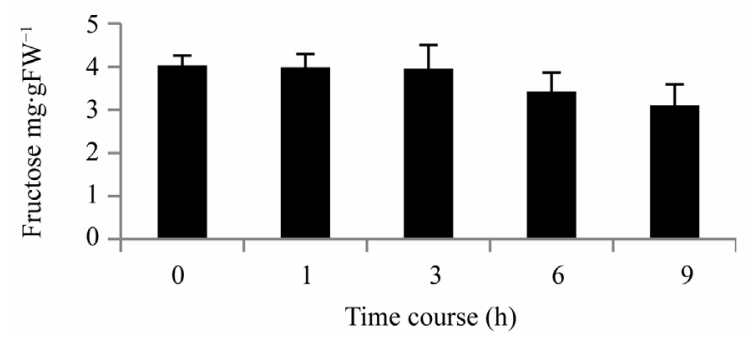

Figure 6. Carbohydrate content profiles for time course experiments in rice seedlings subjected to heat stress. 14-dayold rice seedlings grown at $25^{\circ} \mathrm{C}$ were exposed to $52^{\circ} \mathrm{C}$ to analyze sugar content at $1,3,6$ and $9 \mathrm{~h}$ after the heat treatment; 0h represents control (a) Galactinol content; (b) Sucrose content; (c) Glucose content; (d) Fructose content. Error bar indicated standard error of the mean for three experiments $(\mathrm{n}=3,(*)$ : $\mathrm{p}<0.05,(* *): \mathrm{p}<0.01)$. FW, Fresh Weight. 
treatment on thermotolerance of rice seedlings, we pretreated 14-day-old rice seedlings grown at $25^{\circ} \mathrm{C}$ with 50 $\mathrm{mM}$ raffinose for $12 \mathrm{~h}$ and pre-treated another group of plants with $5 \mu \mathrm{M}$ GDA for $12 \mathrm{~h}$ before subjecting the plants to heat stress at $52^{\circ} \mathrm{C}$ for $3 \mathrm{~h}$. After the heat stress treatment, the rice seedlings were grown under control conditions for 7 days to observe their growth and appearance. The rice seedlings pre-treated with raffinose or GDA remained green and did not show severe leaf injury, whereas the non-treated rice seedlings exhibited severe bleaching after the heat stress (Figures 7(a) and (b)). The difference is particularly clear in enlarged pictures (Figure 6(c)). In combination with the data from our present experiments (Figures 1-6), this suggests that the induction of OsGolS1 expression in response to the GDA treatments or heat stress resulted in the accumulation of galactinol which is converted to raffinose. The intracellular accumulation of these carbohydrates may have acted as osmoprotectants that helped the rice plants cope with severe heat stress.

\section{DISCUSSIONS}

\subsection{The Expressions of OsHsfA2 and OsGolS1 Were Induced under the GDA Treatments in the Absence of Heat Stress}

The accumulated evidence concerning on heat-shock proteins and heat stress signaling in Arabidopsis and other organisms has revealed that; i) under normal conditions, Hsp90 maintains its association with HsfA1 in the cytoplasm, so that HsfA1 remains inactive; ii) Subsequently, under heat stress, the recruitment of Hsp90 by heat-denatured polypeptides interrupts the interaction between Hsp90 and HsfA1, leading to trimerization and subsequent activation of HsfA1; iii) A trimer complex of HsfA1 recognized HSE in promoter regions of several heat-inducible genes as observed under heat stress $[10,18$, 19,21]. Treatment of Arabidopsis plants with GDA or radicicol, which are specific inhibitors for Hsp90, enhanced the expression of heat-inducible genes in the absence of heat stress [18]. In silico expression analysis revealed that a set of Hsf genes class HsfA, HsfB, HsfC families identified in the rice genome are regulated in response to high temperature, drought, and oxidative stress in complex manners [22]. However, the expression-correlation network among those rice Hsfs and putative target genes remains to be clarified.

HsfA1 is considered to be a house keeping gene in plants and a master regulator functioning on heat-induced expression of multiple HSF and HSP genes [1,18]. In the present study, the treatment of the rice seedlings with GDA resulted in the induction of OsHsfA2 and OsGolS1 expression, but the mRNA levels of OsHsfA1 re-
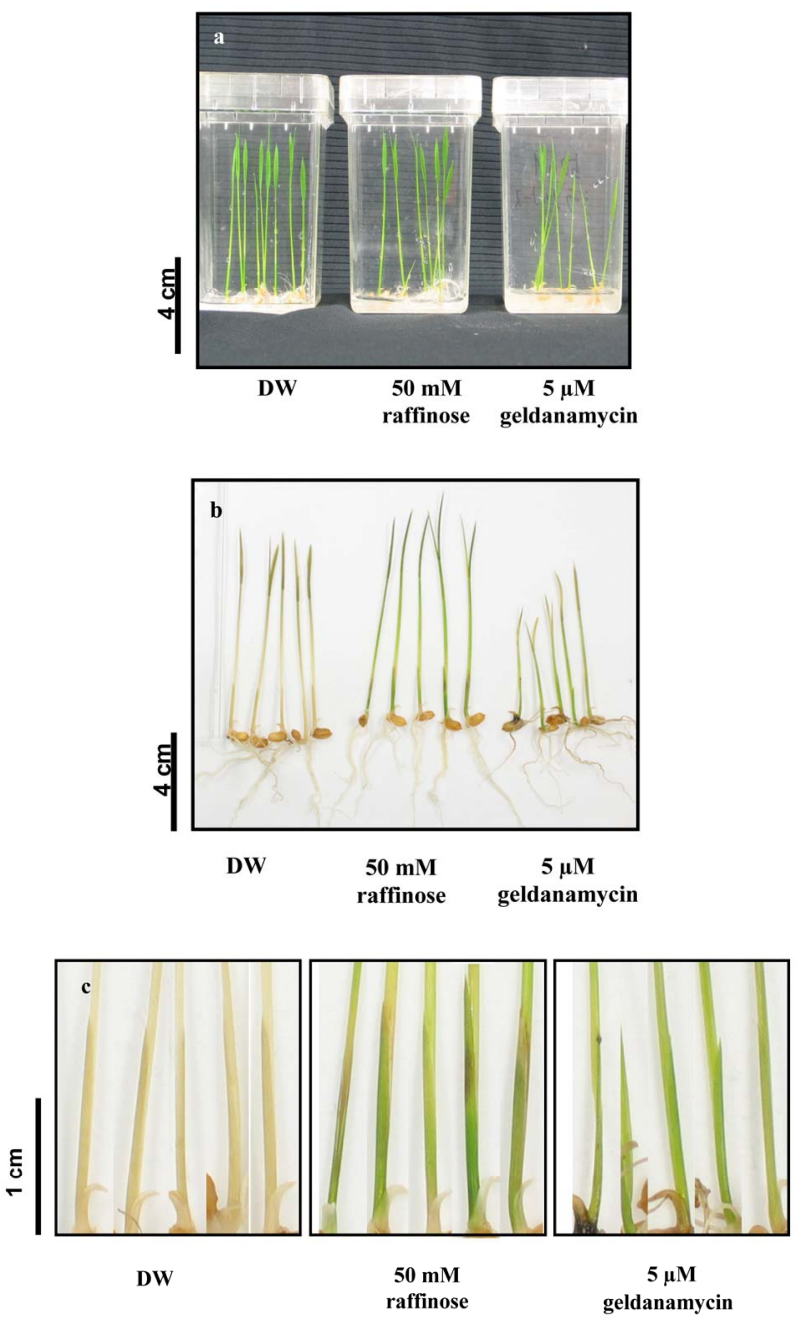

Figure 7. Effects of Raffinose and gendanamycin on appearance of rice seedlings under heat stress. (a) control plants treated by raffinose and by GDA without heat stress; (b) The appearance of rice seedlings pretreated by raffinose or GDA at 7 days after heat stress; (c) Visible color change in leaves of rice seedlings were observed in enlarged pictures. DW: distilled water; $50 \mathrm{mM}$ Raffinose: rice seedlings pre-treated by $50 \mathrm{mM}$ raffinose for $12 \mathrm{~h} ; 5 \mu \mathrm{M}$ geldanamycin: rice seedlings pretreated by $5 \mu \mathrm{M}$ geldanamycin for $12 \mathrm{~h}$.

mained relatively constant (Figure 2). Interestingly, the mRNA level of OsHsfA2 appeared to be induced earlier than that of OsGolS1 in response to the GDA treatment (Figure 2). A study of the stress-responsive cis-element in the putative promoter region (within 1000 bp upstream of the coding region) of AtHsfA2 indicated the presence of HSEs that are recognized by some class A of the AtHsf family [10]. Our study on rice seedlings revealed that OsHsfA2 was up-regulated under both an Hsp90 inhibitor treatment and heat stress conditions (Figures 1, 2). Based on the expression profiles of OsHsfA2 and OsGolS1 under heat stress, it is hypothesized that OsHsfA2 is involved in the up-regulation of Os- 
GolS1 in response to heat stress (Figure 8).

\subsection{OsHsfA1 and OsHsfA2 Are Involved in the Induction of OsGolS1}

Previous studies have revealed that the expression of AtGolS1 and AtGolS2 in Arabidopsis is regulated by AtHsfA1a, AtHsfA1b, and/or AtHsfA2, which is also consistent with the results in transgenic Arabidopsis plants over-expressing AtHsfA2 whose AtGolS1 up-regulation was correlated with AtHsfA2 expression [5,8,9,23]. The GDA treatment on rice seedlings remarkably induced the expression of OsHsfA2, which was followed by the induction of OsGolS1. The gel shift assay revealed that recombinant MBP-OsHsfA2 bound to the promoter region of OsGolS1 containing potent HSE motives, resembling to a strong consensus [nGAAnnTTCnnGAAn], and a number of core consensus motives [nGAAn], [nTTCn]) resulting in the significant mobility shifts (Figures 3(a) and (c)) [19]. In addition, there was no interaction between the recombinant OsHsfA2 and the OsHsfA3 promoter region. It could be expected based on the previous observations that OsHsfA3 is induced under cold stress but not under heat stress $[13,15]$. Furthermore, gel-shift assay revealed that OsHsfA1 binds the promoter regions of OsHsfA2 and OsGolS1 (Figures 4(b) and (c)). In Arabidopsis, HsfAld and HsfAle appeared to be involved in induction of HsfA2, which is a key regulator for aquired tolerance under various environmental stresses [24]. Therefore, it is reasonable that dissociation from the Hsp90-OsHsfA1 under heat stress or GDA treatment activates OsHsfA1 protein, leading to the transcriptional activation of OsHsfA2 and resultant induction of OsGolS1. It can be assumed that a sequential transcription cascade composing of OsHsfA1-OsHsfA2-OsGolS1 play a pivotal role in aquired thermotolerance of rice seedling via galactinol synthesis (Figure 8). Alternatively, it is conceivable that heat stress and ROS differentially induces OsHsfA1 and OsHsfA2, leading to transcriptional activation of OsGolS1 by OsHsfA1-mediated OsHsfA2 induction and or by direct interaction of OsHsfA1 to the OsGolS1 promoter.

\subsection{Galactinol and Raffinose Accumulation Was Correlated with the Expression Levels of OsGolS1 and These Carbohydrate Accumulation Enhanced Thermotolerance}

Recent studies have demonstrated that the metabolic pathways for sucrosyl oligosaccharides, including RFOs and fructans, function in the tolerance of oxidative stress via ROS signaling pathways [25]. It has been reported that the induction of GolS expression in Arabidopsis precedes the accumulation of galactinol, which has com-

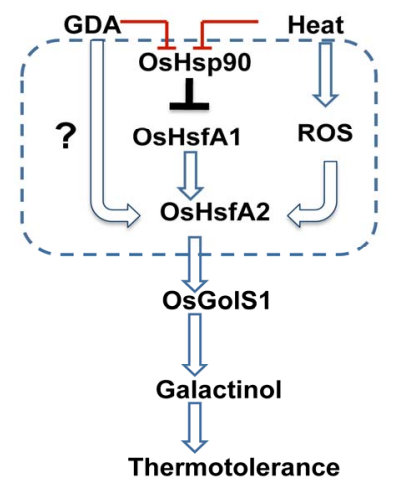

Figure 8. Proposed model of signaling pathway involved in the regulation of OsGolS1 expression under geldanamycin (GDA) and heat stress treatments. OsHsfA2 is up-regulated via OsHsfA1 which is activated by dissociation with OsHsp90 under GDA or heat stress treatments. The up-regulation of $O s H-s f A 2$ could be involved in $O s G o S 1$ induction that is necessary for intracellular accumulation of galactinol. The increasing in carbohydrate levels in plant cells enhances thermotolerance of rice seedlings.

patible solute-like protective abilities, thus reduces the intracellular ROS and oxidant levels [5]. This is consistent with the observation that the $\mathrm{H}_{2} \mathrm{O}_{2}$ contents in rice leaf tissues increased transiently at $3 \mathrm{~h}$ during the heat stress (Figure 5). As the heat stress continued, the $\mathrm{H}_{2} \mathrm{O}_{2}$ contents in the rice leaf tissues decreased to the control level and then remained stable (Figure 4). The expression of OsGolS1 appeared to be highly induced during heat stress and correlated with the accumulation of galactinol (Figures 1 and 5(a)). Raffinose content also increased at approximately as high as the level of galactinol (Figure 6(b)). These results suggest that after the transient increase in intracellular $\mathrm{H}_{2} \mathrm{O}_{2}$, the $\mathrm{H}_{2} \mathrm{O}_{2}$ was consumed by up-regulated scavenging systems such as galactinol and raffinose, which accumulated under heat stress [5]. The reduction of ROS in plant cells enables the plants to cope with high temperature stress. In the present study, we also observed a drastic increase in the content of sucrose, but no significant change in the levels of glucose and fructose under heat stress (Figures 6(c)(e)). Therefore, it can be supposed that together with galactinol and raffinose, sucrose accumulation also plays an important role as an osmoprotectant in the thermotolerance of rice seedlings, even though the increase in sucrose may be regulated in different manner from galactinol. 
It was reported that high temperature treatment on Lycoris enhances oxidative stress leading to acceleration of senescence [26]. The observation that raffinose treatment suppressed early senescence of rice seedling after heat stress indicates that ROS-induced GolS1 plays important roles in aquired tolerance against oxidative damage induced by heat stress (Figures 7). Oxidative damage to the photosynthetic apparatus of Arabidopsis under heat and drought stress appeared to be prevented by over-expression of GolS or by GDA-induced heat-shock proteins $[5,18,27]$. Those previous studies are consistent with our observations that rice seedlings pre-treated with raffinose or GDA showed less damage under heat stress than the controls (Figures 7(b) and (c)). These results indicate that raffinose or GDA treatments can protect photosynthesis apparatus of rice under severe heat stress. It was reported that transgenic Arabidopsis with introduced OsHsfA2 $e$ expressed AtGolS1 at significant levels [28]. The transgenic Arabidpsis overexpressing OsHsfA2e showed enhanced the thermotolerance, similar to those in Arabidopsis with over-expressing AtHsfA2 [5]. Therefore, our results indicate that heat stress or the Hsp90 inhibitor possibly induce the expression of OsHsfA2 by binding of OsHsfA1 to the OsHsfA2 promoter regions, leading to galactinol accumulation by the resultant OsGolS1 induction, and that heat stress- and/or ROS-mediated HsfA2 induction is a critical signal component that exerts the protection mechanisms of plant cells against sub-lethal oxidative stress caused by heat stress (Figure 8).

Increasing evidence suggests that the HSFA2 is a central signal component regulating a set of gene expression essential for the aquired thermotolerance of higher plants. Therefore, identifications and molecular application technique of the signaling molecules at upstream of the HSFA2 are important for breeding of stress-tolerant crops.

\section{ACKNOWLEDGEMENTS}

This research was supported in part by Grant-in-Aids from the Ministry of Education, Sports, Culture, Science and Technology of Japan, No. 23380013 (to Mari Iwaya-Inoue) and by Japan's Grant-in-Aids for Human Resource Development Scholarships (JDS) (to Nong Thi Hue and Huong Thi Tran).

\section{REFERENCES}

[1] Baniwal, S.K., Bharti, K., Chan, K.Y., Fauth, K., Ganguli, A., Kotak, S., Mishra, S.K., Nover, L., Port, M., Scharf, A.D., Tripp, J., Weber, C., Zielinsky, D. and von KoskullDoring, P. (2004) Heat stress response in plants: A complex game with chaperones and more than twenty heat stress transcription factors. Journal of Biosciences, 29, 471-487. doi:10.1007/BF02712120

[2] Lee, B., Won, S. and Lee, H. (2000) Expression of chloroplast-localized small heat shock protein by oxidative stress in rice. Gene, 245, 283-290. doi:10.1016/S0378-1119(00)00043-3

[3] Maestri, E., Klueva, N. and Perrotta, C. (2002) Molecular of heat shock proteins in cereals. Plant Molecular Biology, 48, 667-681. doi:10.1023/A:1014826730024

[4] McLellan, C. and Turbyville, T. (2007) A rhizosphere fungus enhances Arabidopsis thermotolerance through production of an Hsp90 inhibitor. Plant Physiology, 145, 174-182. doi:10.1104/pp.107

[5] Nishizawa, A., Yabuta, Y. and Shigeoka, S. (2008) Galactinol and raffinose constitute a novel function to protect plants from oxidative damage. Plant Physiology, 147, 1251-1263. doi:10.1104/pp.108

[6] Liu, J.-J., Krenz, D.C., Galvez, A.F. and de Lumen, B.D. (1998) Galactinol synthase (GS): Increased enzyme activity and levels of mRNA due to cold and desication. Plant Science, 134, 11-20. doi:10.1016/S0168-9452(98)00042-9

[7] Saravitz, D.M., Pharr, D.M. and Carter, T.E. (1987) Galactinol synthase activity and soluble sugars in developing seeds of four soybean genotypes. Plant Physiology, 83, 185-189. doi:10.1104/pp.83.1.185

[8] Nishizawa, A., Yabuta, Y. and Yoshida, E. (2006) Arabidopsis heat shock transcription factor A2 as a key regulator in response to several types of environmental stress. Plant Journal, 48, 535-547. doi:10.1111/j.1365-313X.2006.02889.x

[9] Nishizawa, A., Tainaka, H., Yoshida, E., Tamoi, M., Yabuta, Y. and Shigeoka, S. (2010) The 26S proteasome function and Hsp90 activity involved in the regulation of HsfA2 expression in response to oxidative stress. Plant and Cell Physiology, 51, 486-496. doi:10.1093/pcp/pcq015

[10] Panikulangara, T.J., Eggers-Schumacher, G. and Wunderlich, M. (2004) Galactinol synthase 1. A novel heat shock factor target gene responsible for heat-induced synthesis of raffinose family oligosaccharides in Arabidopsis. Plant Physiology, 136, 3148-3158. doi:10.1104/pp.104

[11] Phan, T., Ishibashi, Y., Yuasa, T. and Iwaya-Inoue, M. (2010) Chilling stress induces galactinol synthase (OsGolS1) in rice seedlings. Cryobiology and Cryotechnology, 56, 139146.

[12] Yuasa, T., Ishibashi, Y. and Iwaya-Inoue, M. (2012) A flower specific calcineurin B-like molecule (CBL)-interacting protein kinase (CIPK) homolog in yomato cultivar Micro-Tom (Solanum lycopersicum L.). American Journal of Plant Sciences, 3, 753-763. doi:10.4236/ajps.2012.36091

[13] Nakamura, J., Yuasa, T., Huong, T.T., Harano, K., Tanaka, S., Iwata, T., Phan, T. and Iwaya-Inoue, M. (2011) Rice homologs of inducer of CBF expression (OsICE) are involved in cold acclimation. Plant Biotechnology, 28, $303-$ 309. doi:10.5511/plantbiotechnology.11.0421a

[14] Storozhenko, S., Pauw, P.D., Motagu, M.V., Inze, D. and Kushnir, S. (1998) The heat-shock element is a functional component of the Arabidopsis APX1 gene promoter. Plant Physiology, 118, 1005-1014. doi:10.1104/pp.118.3.1005

[15] Liu, A.L., Zou, J., Zhang, X.W., Wang, W.F., Xiong, X.Y., 
Chen, L.Y. and Chen, X.B. (2010) Expression profiles of class A rice heat shock transcription factor genes under abiotic stresses. Journal of Plant Biology, 53, 142-149. doi:10.1007/s12374-010-9099-6

[16] Veljovic-Jovanovic, S., Noctor, G. and Foyer, C.H. (2002) Are leaf hydrogen peroxide concentrations commonly overestimated? The potential influence of artefactual interference by tissue phenolics and ascorbate. Plant Physiology and Biochemistry, 40, 501-507. doi:10.1016/S0981-9428(02)01417-1

[17] Ishibashi, Y., Yamaguchi, H., Yuasa, T., Iwaya-Inoue, M., Arima, S. and Zheng, S.H. (2011) Hydrogen peroxide spraying alleviates drought stress in soybean plants. Journal of Plant Physiology, 168, 1562-1567. doi:10.1016/j.jplph.2011.02.003

[18] Yamada, K., Uchida, A., Fukao, Y., Hayashi, M., Fukazawa, M., Suzuki, I. and Nishimura, M. (2007) Cytosolic HSP90 regulates the heat shock response that is responsible for heat acclimation in Arabidopsis thaliana. Journal of Biological Chemistry, 282, 37794-37804. doi:10.1074/jbc.M707168200

[19] Schoffl, F., Prandl, R. and Reindl, A. (1998) Regulation of the heat-shock response. Plant Physiology, 117, 11351141. doi:10.1104/pp.117.4.1135

[20] Asada, K. (2006) Production and scavenging of reactive oxygen species in chloroplasts and their functions. Plant Physiology, 141, 391-396. doi:10.1104/pp.106.082040

[21] Kotak, S., Larkindale, J., Lee, U., von Koskull-Doring, P., Vierling, E. and Scharf, K.D. (2007) Complexity of the heat stress responses in plants. Current Opinion in Plant Biology, 10, 310-316.doi:10.1016/j.pbi.2007.04.011

[22] Mittal, D., Chakrabarti, S., Sarkara, A., Singh, A. and Grover, A. (2009) Heat shock factor gene family in rice: Genomic organization and transcript expression profiling in response to high temperature, low temperature and oxidative stresses. Plant Physiology and Biochemistry, 47,
785-795. doi:10.1016/j.plaphy.2009.05.003

[23] Nishizawa, A., Yoshida, E., Yabuta, Y. and Shigeoka, S. (2009) Analysis of the regulation of target gene by and Arabidopsis heat shock transcription factor, HsfA2. Bioscience, Biothechnology, and Biochemistry, 73, 890-895. doi:10.1271/bbb.80809

[24] Nishizawa-Yokoi, A., Nosaka, R., Hayashi, H., Tainaka, H., Maruta, T., Tamoi, M., Ikeda, M., Ohme-Takagi, M., Yoshimura, K., Yabuta, Y. and Shigeoka, S. (2011) HsfA1d and HsfAle involved in the transcriptional regulation of HsfA2 function as key regulators for the Hsf signaling network in response to environmental stress. Plant and Cell Physiology, 52, 933-945.

doi: $10.1093 / \mathrm{pcp} / \mathrm{pcr} 045$

[25] Van den Ende, W. and Valluru, R. (2009) Sucrose, sucrosyl oligosaccharides, and oxidative stress: Scavenging and salvaging? Journal of Experimental Botany, 60, 9-18. doi:10.1093/jxb/ern297

[26] Boonyaritthongchai, P., Manuwong, S., Kanlayanarat, S., Nakamura, Y., Okamoto, S. and Matsuo, T. (2008) Acceleration of senescence by high temperature treatment in Lycoris (L. traubii $\times$ L. sanguinea) leaf sections. Journal of the Japanese Society for Horticulture Science, 77, 431439. doi:10.2503/jjshs1.77.431

[27] Taji, T., Ohsumi, C., Iuchi, S., Seki, M., Kasuga, M., Kobayashi, M., Yamaguchi-Shinozaki, K. and Shinozaki, K. (2002) Important roles of drought- and cold-inducible genes for galactinol synthase in stress tolerance in Arabidopsis thaliana. Plant Journal, 29, 417-426. doi:10.1046/j.0960-7412.2001.01227.x

[28] Yokotani, N., Ichikawa, T., Kondou, Y., Matsui, M., Hirochika, H., Iwabuchi, M. and Oda, K. (2008) Expression of rice heat stress transcription factor OsHsfA2e enhances tolerance to environmental stresses in transgenic Arabidopsis. Planta, 227, 957-967. doi:10.1007/s00425-007-0670-4 